\title{
\begin{tabular}{l|l} 
pcori $).$ & PATIENT-CENTERED OUTCOMES RESEARCH INSTITUTE \\
RESEARCH SUMMARY
\end{tabular}
}

\section{Matching Patients with Therapists to Improve Mental Health Care}

Principal investigator

Michael J. Constantino, PhD
Organization

University of Massachusetts Amherst

\section{What was the research about?}

Therapy is a common treatment for many mental health concerns. Some therapists do better at treating certain concerns, such as depression or substance use, than others. But mental health centers don't often consider how well therapists treat a certain concern when they assign patients to therapists. Instead, they may assign patients based on practical things like which therapist has an available appointment.

In this study, the research team tested a system called Match. Match pairs patients with therapists based on how well a therapist has treated patients with the same concerns in the past. The research team compared patients using Match with those assigned to a therapist using a clinic's usual approach. Neither patients nor therapists were aware of how they were paired.

\section{What were the results?}

Across four months, compared with patients not paired with a therapist using Match, patients paired using Match

- Had greater reduction in general mental health symptoms, impaired functioning, and symptoms specific to their mental health concerns

- Had more consistent treatment results

- Felt less distressed or upset

Patients paired with a therapist using and not using Match didn't differ in
- The quality of their relationship with their therapist

- How well they expected treatment to work

- Whether they stopped treatment early

- How satisfied they were with their therapist

\section{Who was in the study?}

The study included 218 patients who were referred to mental health care. Of these patients, 89 percent were White, 6 percent were Black, 3 percent were Latino, and 2 percent were another race. The average age was 34 , and 67 percent were women. All received care at one of six mental health centers in Cleveland, Ohio.

\section{What did the research team do?}

The research team assigned patients by chance to one of two groups. In the first group, the mental health center used Match to pair patients with therapists. Match used information from at least 15 past patients to rate therapists as effective, neutral, or ineffective for each of 12 mental health concerns. Then the mental health center assigned each patient in the study to a therapist who had been rated as effective for their concerns. In the second group, the mental health center assigned patients to therapists in their usual way. All therapists treated patients as they would have if they weren't in the study.

Patients filled out surveys before treatment started and multiple times during their four months of treatment. 
Patients, therapists, referring doctors, and mental health center staff provided input during the study.

\section{What were the limits of the study?}

The research team tested Match in one health system in the Midwest; results might differ in other places. To rate therapists for certain concerns, the team looked at only one measure of their performance with past patients. Ratings might differ with other measures.
Future research could test Match in other places or use other measures to rate therapists' past performance.

\section{How can people use the results?}

Mental health centers can use these results when considering how to pair patients with therapists to improve mental health care.

To learn more about this project, visit www.pcori.org/Constantino376. 